\title{
Efecto blanqueador significativo de cinco pastas dentales blanqueadoras
}

Significative whitening action of five whitening toothpastes

\begin{abstract}
Resumen
Una principal preocupación de los pacientes es mejorar la estética dental mediante el blanqueamiento, por ello existen productos como pastas dentales blanqueadoras que intentan satisfacer esa necesidad. Para confirmar el efecto clínico de blanqueamiento de cinco pastas dentales con esta propiedad en piezas dentales anteriores, se estructuraron cinco grupos experimentales para cada pasta, conformadas por 5 participantes por grupo, de 18 a 25 años de edad y un grupo control. El color fue evaluado con un colorimetro de blanqueamiento VITA mediante observación directa y lectura final a las 7 semanas. Se calibró la técnica de cepillado y tipo de cepillo. Se observó una variación leve del color para todas las pastas dentales tanto las experimentales como el control. Se encontró diferencias significativas de cambio de color del pre y post evaluación en dos pastas dentales (Colgate Maxwhite y Crest Peroxide); con un $\mathrm{p}=0,039$ para ambos. No se obtuvieron diferencias significativas para los colores finales entre las pastas dentales blanqueadoras y el control $(p=0,549)$. Se concluye que las pastas dentales utilizadas produjeron cambio de coloración en los dientes analizados. Solo las pastas Colgate Maxwhite y Crest Peroxide produjeron efecto blanqueador significativo en la variación del color inicial y final. Sin embargo, no hubo un efecto blanqueador real entre pastas experimentales y el control.
\end{abstract}

\begin{abstract}
One of the main concerns of patients is to improve their dental aesthetics through teeth whitening, which is why there is a big quantity of products in the market that tries to satisfy this requirement with whitening toothpastes offer.

To determinate whitening clinic effect of 5 toothpastes in dental teeth, they were arranged 5 treatment groups for each toothpaste conformed for 5 students and a control group. Subjects were between 18 and 25 years. Colour was evaluated with Vita Bleaching Shade Guide at the beginning and after 7 weeks through direct observation. Subjects were instructed to brush their teeth using the same toothbrush and the same technique too. There were few changes of colour for toothpaste used, in the experimental as the control group.It was found statistically significant changes of colour between pre and post test in 2 toothpastes (Colgate Max White and Crest Peroxide) $(p=0.035)$. There was not obtained significant differences between whitening toothpaste and control group $(p=0.549)$ As a conclusion, toothpastes used ( 5 whitening paste and one control paste) made changes on the teeth analyzed, only Colgate Max White y Crest Peroxide produce a significant whitening action on the colour variation. However there was not found a real whitening action between experimental and control toothpaste.
\end{abstract}

\section{Introducción}

Para las personas es muy importante, la forma, tamaño y color de sus dientes. Se busca tener dientes más blancos, que reflejen un aparente estado de higiene bucal óptima y una sonrisa estéticamente agradable. En la mayoría de los casos la aplicación de cualquier agente blanqueador se debe a la presencia de pigmentaciones o manchas clasificadas en dos categorías: extrínsecas e intrínsecas.' Las primeras son fácilmente eliminadas y ocurren cuando algún agente externo mancha o daña la superficie de los dientes. Los factores extrínsecos tabaco, cigarros y pipas provocaran oscurecimiento de los tonos amarillo-marrón a negro, en general en la parte cervical de los dientes $y$, de forma primaria, en las superficies internas. Las manchas de marihuana se manifiestan por tonos marrón oscuro a negro, en general, en forma de anillos bien destacados alrededor de la parte cervical de los dientes, cerca del margen de la gingiva. El hábito de masticar tabaco provoca manchas que en general penetran en el esmalte de forma más profunda; ya el café y el té causan oscurecimiento más severo y tenaz, en general de tono marrón a negro. ${ }^{1}$ Las manchas intrínsecas están localizadas dentro de la estructura dental y son de pronóstico menos favorable para cualquier tipo de blanqueamiento. ${ }^{1}$

\section{Margot Gutiérrez llave, 'Luis Bernuy Torres, ${ }^{2}$ Katia Medina Calderón, ${ }^{1}$ Grisel Vadillo Palacios. ${ }^{2}$}

\author{
Departamento Académico Biosocial \\ 2 Practica Privada. \\ Correpondencia: \\ Margot Margarita Gutiérrez llave
}

Facultad de Odontología. UNMSM. Av. Germán Amézaga s/n. Lima 1. Perú

Teléfono: 996466678

Correo electrónico: margoti11@yahoo.es

Palabras Clave: Color de dientes. Efecto blanqueador. Pasta dental.

Key words: Whitening action; toothpastes; dental colour.

La función principal de un dentífrico es limpiar y pulir las superficies de fácil acceso de los dientes cuando se utiliza en combinación con un cepillo de dientes y debe proporcionar la limpieza máxima con la menor abrasión posible de los tejidos dentales.

La abrasión que ocurre durante el uso de cualquier agente pulidor dentífrico actúa mediante una función de: la dureza inherente del material abrasivo, el tamaño de la partícula y la forma del producto fresador; las propiedades de la mezcla abrasiva ( $\mathrm{pH}$, viscosidad, conductividad de calor), la dureza de las cerdas; la fuerza que se aplique durante el cepillado, las propiedades de la superficie que sufre la abrasión, 
por ejemplo, esmalte o dentina. Actualmente se ofrecen en el mercado diversas pastas dentales con efecto blanqueador, por lo que el paciente aplicándose la pasta dental durante su higiene diaria bucal, lograría "blanquear" progresivamente sus dientes luego de un periodo de tiempo de utilizado el producto., ${ }^{2,3}$ Bajo esta premisa se han realizado estudios sobre el efecto blanqueador de estas pastas comerciales. Terézhalmy y $\mathrm{col}^{4,5}$ en un estudio sobre la remoción de manchas dentales mediante la aplicación de un dentífrico blanqueador y la combinación de otros dos por un período de dos semanas en adultos de 22 a 30 años, observaron una reducción significativa, sin embargo, no encontraron diferencias entre ambos grupos experimentales. He y $\mathrm{col}^{6} \mathrm{Compararon}$ la combinación de fluoruro estañoso y hexametafosfato de sodio, con la acción de una pasta dental blanqueadora en la reducción de manchas, observando una reducción significativa al cabo de 6 semanas para ambos grupos experimentales, sin encontrar diferencias entre ambas sustancias.

Se entiende como efecto blanqueador al cambio de coloración del diente mediante la aplicación de un agente blanqueador manifestado hacia un estado de clareamiento. Para ello se debe tener presente el concepto de color que abarca no solo el estímulo electromagnético, sino también la recepción de este estímulo por el ojo y de la interpretación de los mismos por el cerebro. ${ }^{7}$ Por esto los investigadores, no consideran el color sólo como un fenómeno físico, sino como una asociación entre lo físico y lo psíquico, dependiendo de la interpretación del individuo receptor, lo que está directamente asociado a sus conocimientos previos en un nivel consciente $\mathrm{e}$ inconsciente. ${ }^{8-10}$

El objetivo fue determinar el efecto clínico blanqueador real de las pastas dentales blanqueadoras comerciales en dos tipos de piezas dentales anteriores (Canino superior derecho / incisivo central superior derecho) en un grupo de estudiantes de la Facultad de Odontología de la UNMSM.

\section{Materiales y método}

El estudio cuasi - experimental, se realizó en una muestra no probabilística selečcionada por conveniencia de 30 estudiantes de los cuales 16 correspondían al género femenino y 14 al género masculino. Se distibuyeron en cinco grupos experimentales y un grupo control o testigo, conformado por cinco estudiantes por grupo. Las pastas dentales experimentales fueron: Oral Fresh, Aquafresh, Colgate Max White, Colgate Total, Crest Peroxido, y el grupo control utilizó una pasta dental convencional (Dento).

\section{Procedimientos y técnicas}

Para la adquisición de pastas dentales blanqueadoras el criterio de selección fue que en el empaque manifestara ser "blanqueadora" y que indujera al comprador al cambio en la tonalidad natural del color de sus dientes; no se consideró a las pastas que a pesar de tener el titulo de blanqueadoras en sus especificaciones indicaran que solo devolverían el color natural de los dientes del consumidor.

La pasta dental control fue de la marca Dento que en su empaque no manifiestan la propiedad de ser blanqueadora.

Previamente a la toma de datos, a cada participante, se les entregó una cartilla de instrucciones sobre la técnica de cepillado a utilizar, frecuencia de cepillado, tiempo de cepillado, cantidad de pasta dental a utilizar y algunas restricciones como: limitar el consumo de bebidas con colorantes, no consumir café ni fumar. Además se les hizo una demostración práctica de la técnica de cepillado de Bass modificado con la ayuda de un tipodont y un cepillo. Igualmente se le hizo entrega de una pasta dental (blanqueadora para los grupos experimentales y convencional para el grupo control) un cepillo de dientes y una cartilla de instrucciones. El día del registro de datos, se indicó a los participantes que realicen un cepillado dental previo con la técnica demostrada; en el caso de las damas sin pintura de labios.

Para la toma de la medida de color se buscó un lugar adecuado, escogiéndose un ambiente cerrado con luz artificial blanca de tipo fluorescente. Se comparó dos piezas específicamente con las tonalidades del colorímetro: el incisivo central superior y el canino superior derechos. El operador se acercó a $60 \mathrm{~cm}$ de la persona y comparó solo una tonalidad del colorímetro por vez a la estructura dentaria. No se acercó todo el colorímetro. La duración de la observación no duró más de 5 segundos en ningún caso, con ello se evitó el cansancio de la retina del operador y afectar la toma de color. Se registró en la ficha de datos y se procedió a tomar fotografías de las piezas observadas, con una cámara fotográfica de 10 mega píxeles. Las observaciones del posible cambio de color se realizaron a las 7 semanas siguiendo las mismas indicaciones.

\section{Resultados}

En la observación inicial para los incisivos centrales se èncontró que el $35 \%$ tuvo un color 2,5 M2 según la clasificación del colorímetro para blanqueamiento VITA Bleachedguide 3M Master y el más del $20 \%$ el color 3M2. (Fig 1).

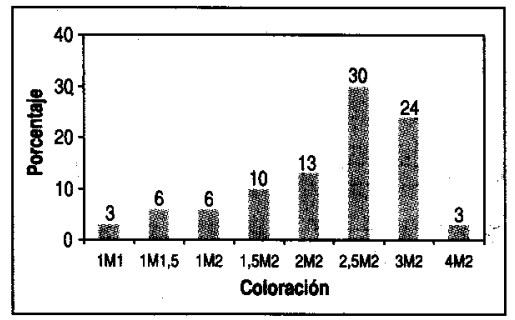

Fig. 1. Coloración inicial de los incisivos centrales.

Para las coloraciones de los caninos superiores se encontro predominantemente los tipos 3M2 y 3,5M2.

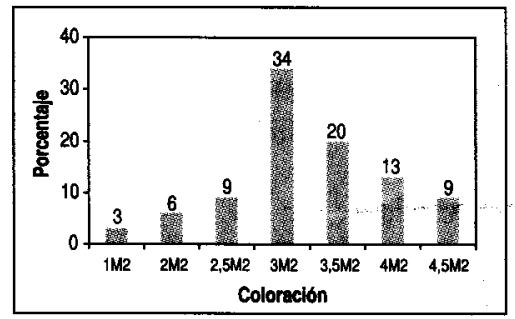

Fig. 2. Coloración inicial de los caninos superiores.

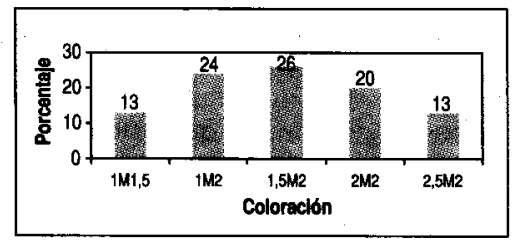

Fig. 3. Coloración de los incisivos centrales superiores.

En la observación del color, después de 7 semanas de tratamiento, se halló que los incisivos centrales presentaron el color 1,5M2 (más de $25 \%$ ) y $1 \mathrm{M} 2$ (más del 20\%) (Fig. 3)

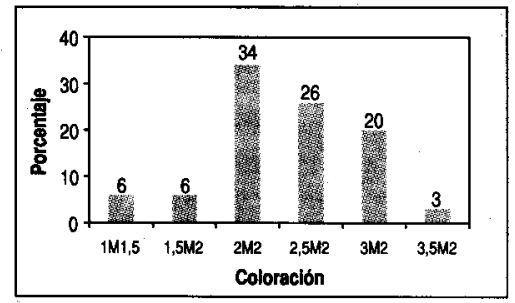

Fig .4. Coloración final de caninos superiores. 
Mientras que en la observación final del color de los caninos superiores se encontró el color $2 \mathrm{M} 2$ (más del $30 \%$ ) y 2,5M2 (más el 25\%) (Fig. 4).

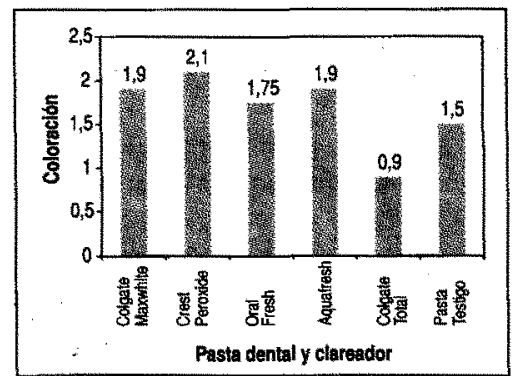

Fig. 5. Variabilidad de los colores de los incisivos centrales.

Al realizar la comparación entre los colores iniciales y finales, se observa una variación leve del color para todas las pastas utilizadas, tanto para las blanqueadoras como para el control. Esta diferencia no es significativa $(p>0,05)$ según la Prueba Kruskal Wallis. Fig. 5

Para la evaluación individual de cada pasta, se aplicó la Prueba de Wilcoxon, encontrándose que para Colgate Maxwhite hubo un $p=0,039$ en los incisivos centrales superiores derechos y un $p=0,041$ para los caninos superiores derechos y para Crest Peroxide hubo un $p=0,059$ en los incisivos centrales superiores derechos y un $p=0,039$ para los caninos superiores derechos.

\section{Discusion}

Para todas las piezas dentales que fueron expuestas a las pastas dentales blanqueadoras se encontró una variación de color sin embargo no hubo diferencia significativa entre las pastas aplicadas del grupo experimental y las del grupo control.

Se podria determinar que estos efectos fueron logrados debido a la propiedad abrasiva de todas las pastas dentales utilizadas demostrado en el estudio realizado por Vicentini y col (2007) ${ }^{3}$ en el que las pastas dentales blanqueadores ejercen un efecto abrasivo a nivel de dentina. Sin embargo, en dos investigaciones para determinar el poder de aclaración de las pastas dentales blanqueadoras sobre manchas presentes en las caras superficiales de los dientes anteriores, los resultados arrojaron una diferencia significativa en un periodo de 2 semanas., 4,511

En los resultados establecidos en el presente estudio, se observó una variación leve del color para todas las pastas, tanto para las pastas blanqueadoras como para las pastas convencionales utilizadas.

Dentro del grupo experimental, las pastas blanqueadoras, que ejercieron un efecto blanqueador significativo, destacaron: Colgate Maxwhite con un índice alto en los incisivos centrales superiores derechos y caninos superiores derechos y para las pastas dentales Crest Peroxide que alcanzó un índice relativamente alto comparado con la pasta anterior.

\section{Conclusiones}

Las pastas dentales utilizadas (5 pastas blanqueadoras y 1 pasta convencional) produjeron cambio de coloración en los dientes analizados. No se obtuvo diferencias significativas al analizar los colores finales entre las pastas dentales blanqueadoras y la pasta dental convencional. lis probable que el cambio de color logrado no necesariamente se deba al efecto blanqueador sino al proceso mecánico de abrasión y/o clareamiento de la superficie dentaria.

Solo las pastas Colgate Maxwhite y Crest Peroxide produjeron un efecto blanqueador significativo en la variación del color inicial y final de las piezas dentarias evaluadas. Lo que sugiere que estas pastas dentales sí lograrían un efecto blanqueador real.

\section{Agradecimiento:}

A los Estudiantes de la Facultad de Odontología de la Universidad Nacional Mayor San Marcos: Mariela Calderón Valencia, Óscar Barreda Torres, José Acha Sinche, Patricia Aguirre Montes, Gracely Ayala González, Cinthia Berrocal Medrano y William Chirri Trebejos por su participación activa en el estudio.

\section{Referencias bib!lográficas}

1. Alves $C$, Rielson J. Estética Odontológica. Sao Paulo, Brasil: Fd. Nueva Generación, 2003: 98-103.
2. Lozada $O$, García C. Riesgos y beneficios del blanqueamiento dental. Home Ediciones, 2000.Pág 57-72.

3. Vicentini $B C$, Braga $S R$, Sobral MA. Medición in vitro de la abrasión de los dentifricos en la dentina. Int Dent $J$., 2007; 57(5):314-318

4. Terezhalmy GT, Biesbroock AR, Farrell S, Barker ML, Bartizec RD. Blanqueamiento dental mediante la eliminación de manchas extrínsecas con dos hexametafosfato de sodio que contienen los dentífricos blanqueadores. Am J Dent, 2007; 20(5):309-314.

5. Terezhalmy $G$, Chaves E, Bsoul S, Baker $\mathrm{R}, \mathrm{He} \mathrm{T}$. Evaluación clínica de la eficacia en la remoción de manchas de una película con un dentífrico fluoruro estañoso con hexametafosfato de sodio. Am J Dent 2007; 20(1): 53-58.

6. He T, Baker R, Bartizek RD, Biesdbrok $A R$, Chaves E, Terezhalmy G. Eficacia de la remoción de manchas extrínsecas de un dentífrico de fluoruro estañoso con hexametafosfato de sodio. Clin Dent, 2007; 18(1):7-11.

7. Melo N, Gallego G, Restrepo L, Pelaez A. Blanqueamiento vital y métodos para la valoración de su eficacia y estabilidad. Revista CES 2006; 19(2):67-69.

8. Lath DL, Smith RN, Guan YH, Karmo M, Brook AH. Medición de mancha en dientes extraídos mediante espectrofotometría y análisis digital de imágenes. Int J Dent Hyg 2007; 5(3):174-179.

9. LuoW, Wetland S, Bruntoon P, Ellwood R, Pretty IA, Mohan N. Comparación de la capacidad de los diferentes índices de color para evaluar los cambios en la blancura de dientes. J Dent 2007; 35(2):109-116.

10. Amengual J, Llena $C$, Forner L. Reproducibilidad en la medición del color in Vitro e in Vivo mediante colorimetros específicos para uso dental. RCOE 2005; 10(3):9-13.

11. Taboada O, Cortes L, Cortes R. Eficacia del tratamiento combinado de peróxido de carbamida al $35 \%$ y $10 \%$ como material blanqueador en flucirosis dental. Revista ADM 2002; 59(3):81-86.

Fecha de recepción: 15 abril 2009

Fecha de aceptación: 15 julio 2009 